\title{
DIGITAL WORKFLOW FOR VIRTUALLY DESIGNING AND PRESSING ULTRA-THIN LITHIUM-DISILICATE VENEERS FOR ESTHETIC REHABILITATION OF FRACTURED MAXILLARY INCISORS - A CASE REPORT
}

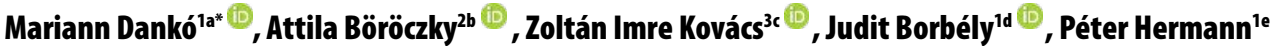

'Department of Prosthodontics, Faculty of Dentistry, Semmelweis University, Budapest, Hungary

IIndependent Óvár Dental Technology Kkt., Hungary

${ }^{3}$ Department of General Dental Preclinical Practice, Faculty of Dentistry, Semmelweis University, Budapest, Hungary

aDMD, Resident

bT

DMD

dDMD, PhD

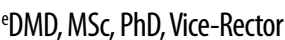

\section{ABSTRACT \\ DOI: https://doi.org/10.25241/stomaeduj.2019.6(4).art.6}

Aim: The aim of this case report is to explain the clinical and laboratory procedures for fabricating ultra-thin lithium-disilicate pressed veneers using a mixed analog/ digital workflow.

Summary: Four ultra-thin pressed lithium-disilicate veneers were produced for maxillary fractured incisors with the help of virtual smile design, digital wax-up and intraoral mock-up. With initial photos from the patient, the smile design could be performed considering the patient's individual face form. An intraoral scan was taken for the digital wax-up, which was guided by the contour line of smile design on the face photo. A printed model and template were manufactured for the mock-up. After a guided minimally invasive preparation and digital impression, the final veneers were designed as an exact copy of the mock-up. Wax pattern was designed on computer and fabricated by milling machine. Finally, lithiumdisilicate veneers were pressed, individualized and cemented using an adhesive technique.

Key learning points: 1 . digital workflow is convenient for a predictable procedure making ultra-thin lithium-disilicate pressed veneers. 2. the virtual smile design digital wax-up - intraoral mock-up scheme presents an opportunity for effective communication with the patient and laboratory. 3. smile design software recommends an ideal smile curve for the patient with the help of definitive points and reference lines on the face. 4. guided preparation through mock-up preserves the greatest amount of enamel structure, which is important with regards to adhesive cementation. 5. when pressing lithium-disilicate veneers, a mixed analog/digital workflow using CAD and a milled wax pattern results in better physical properties and marginal fit than fully digitally milled veneers.

Keywords: Smile Design; Digital Impression; Lithium-Disilicate; Veneers;

Pressed Veneers; Adhesive Cementation.

\section{Introduction}

Working with ceramic restorations in the anterior region is still a great challenge. The number and variety of ceramic materials are increasing, offering thinner wall thicknesses with better physical and optical properties and a wide range of options when a patient needs an esthetic rehabilitation of the maxillary anterior teeth and smile. On the other hand, the patients' expectations are growing regarding not only the esthetics of the restoration

\section{OOPEN ACCESS This is an Open Access article under the CC BY-NC 4.0 license. \\ IsPeer-Reviewed Article \\ Citation: Dankó M, Böröczky A, Kovács Zl, Borbély J, Hermann P. Digital workflow for virtually designing and pressing ultra-thin lithium-disilicate veneers for esthetic rehabilitation of fractured maxillary incisors - a case report. Stoma Edu J. 2019;6(4):261-270 \\ Received: November 05, 2019 Revised December 05, 2019 Accepted: December 12, 2019 Published: December 17, 2019 \\ *Corresponding author: \\ Department of Prosthodontis, Faculty of Dentistry, Semmelweis University Street 47, 1088 Budapest, Hungary el/Fax: +36703643723 e-mail: dankomariann56@gmail.com \\ Copyright: $\odot 2019$ \\ the Editorial Council for the Stomatology Edu Journal.}

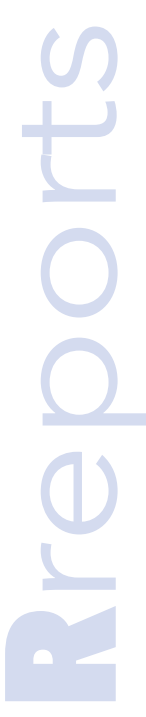




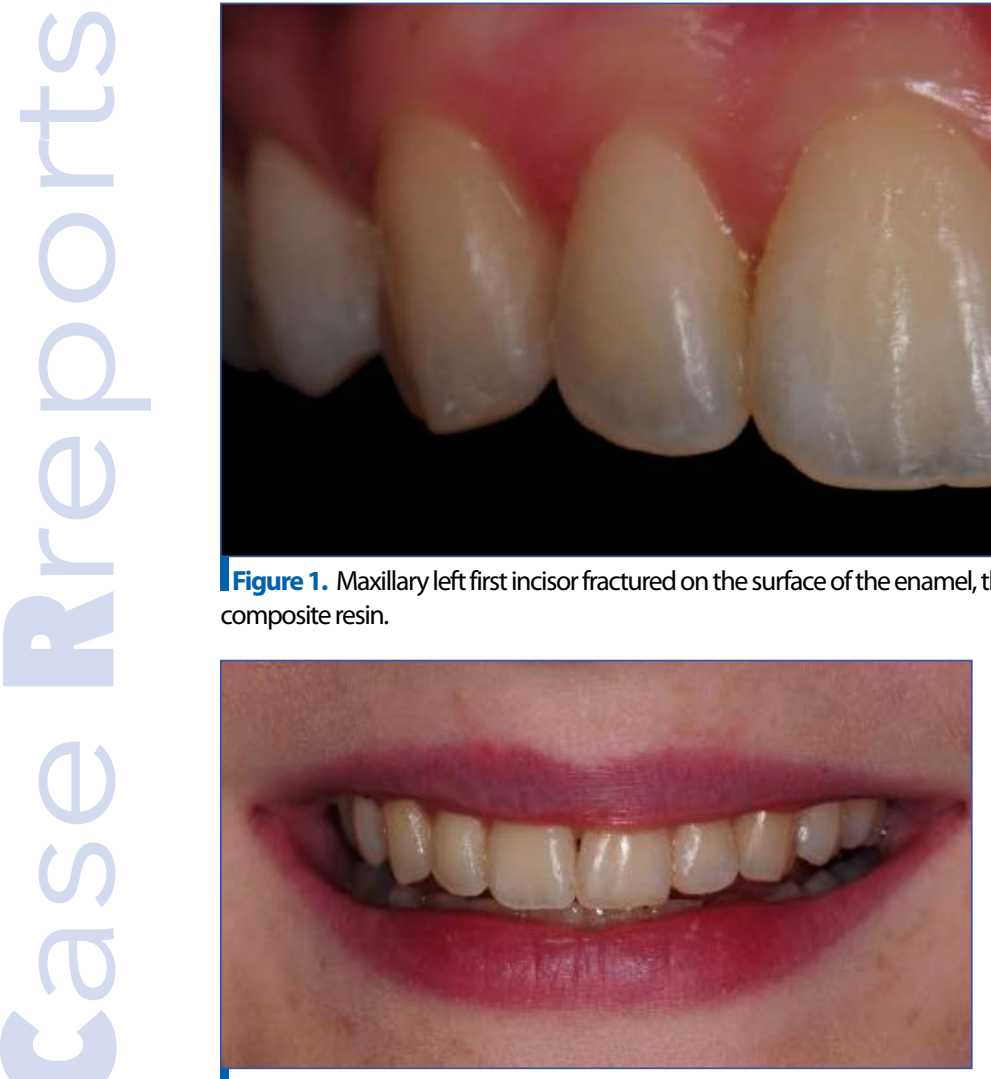

Figure 2. Initial smile photo.

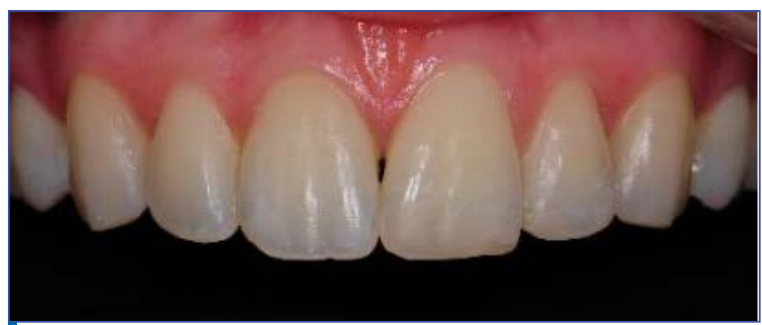

Figure 4. Bleached teeth after 6 weeks

invasive veneers $[4,5]$. The indications for these restorations include: discolored teeth after root canal treatment, tetracyclin, hypoplasia, hypercalcification or fluorosis; covering small composite restorations; closing small diastemas; completing fractured teeth or enamel chipping; altering the shape of teeth; and correcting minor orthodontic problems such as rotation [6].

A suitable material for this application is lithiumdisilicate, which can be processed either by conventional pressing or using digital milling techniques. Both are indicated for manufacturing ultra-thin veneers; however, pressed lithiumdisilicate wall thicknesses can be reduced by an extra $0.1 \mathrm{~mm}$. The minimum wall thickness for the pressed variant is $0.3 \mathrm{~mm}$ in the gingival third and $0.4 \mathrm{~mm}$ in the mid and incisal parts, while for the milled variant it is 0.4 and $0.5 \mathrm{~mm}$, respectively [7]. The Scientific Documentation by Ivoclar Vivadent $[8,9]$ and other recent studies $[10,11]$ showed that the fracture toughness of the pressed material is higher than that of the milled solution. According to Azar et al [12] the marginal fit of the pressed

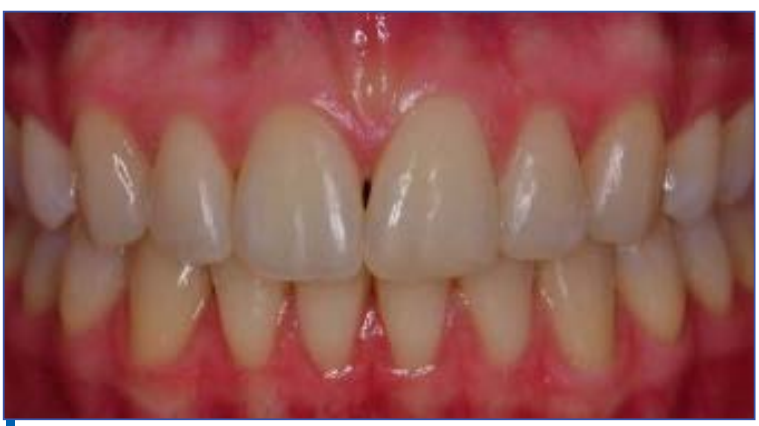

Figure 3. Initial maximal intercuspation position.

restorations is also better than milled. Overall, digital clinical and laboratory workflow is important because of the possibility it provides to design and show a new smile to the patient, and to achieve this form in the final restoration. When an impression of the prepared abutments is taken with an intraoral scanner, full digital workflow can be used with the advantage of time-efficiency, reduced discomfort for the patient, simplified clinical procedures, and better communication with the laboratory and the patient, given the trueness and precision levels comparable to conventional impressions [13].

This fully digital method to mill from a lithiumdisilicate block would be faster. Yet, despite the minimally invasive case in this study, the conventional pressing technique combined with digital workflow was preferred, because of the mentioned superior physical properties, more accurate marginal fit and mainly the reducible wall thickness of pressed lithium-disilicate restorations. This case report aims to explain the clinical and laboratory procedures for fabricating ultra-thin lithium-disilicate pressed veneers using a mixed analog/digital workflow.

\section{Case report}

2.1. Treatment plan

A 25-year-old female patient presented with a need for esthetic rehabilitation of her fractured incisors after a bicycle accident and improvement to the appearance of her smile. The mesial incisal edge of the maxillary left lateral incisor had been missing since the accident and was completed with composite 


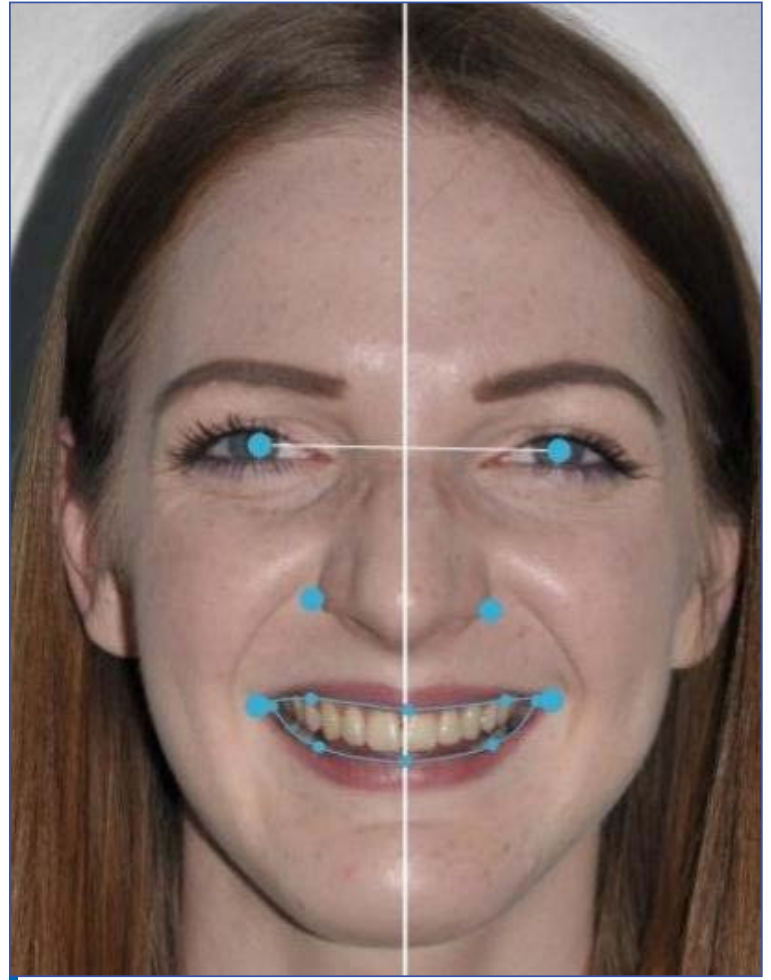

Figure 5. Imported photo and reference points in smile design program.

material as a temporary solution for 2 months. The right and left central incisors were only fractured on the surface of the enamel, but some discoloration had appeared on the left central incisor (Figs. 1-3). The treatment plan was to bleach the teeth on both arches, then to prepare her four maxillary incisors with a minimally invasive method for ultra-thin lithium-disilicate pressed veneers, which she agreed to after being informed about the benefits and risks of the treatment. The bleaching was performed by 40\% hydrogen peroxide (Opalescence Boost 40\%, Ultradent Products, South Jordan, UT, USA) and blue-light whitening accelerator lamp (Beyond Command Whitening Accelerator, Beyond European Headquarters, Zielona Gora, Poland), which resulted in B1 color (Fig. 4).

\subsection{Smile design}

A virtual smile design and an intraoral mock-up constitute the best way to show the patient the designed form of her teeth and the appearance of a new smile. It is essential that this method should give the opportunity to change and correct the form according to the patient's expectations before producing the final veneers. First, a full-face smile photo and a retracted frontal photo were needed for the smile design. After importing the photos in the smile design program (Smile Design, 3Shape, Copenhagen, Denmark), the following reference points and lines were set: pupils, nasal wings, and curve of the lips. In the next step, the two photos were aligned moving two pairs of points (Figs. 5-6). According to the reference points, the software recommended an ideal gingival and smile curve

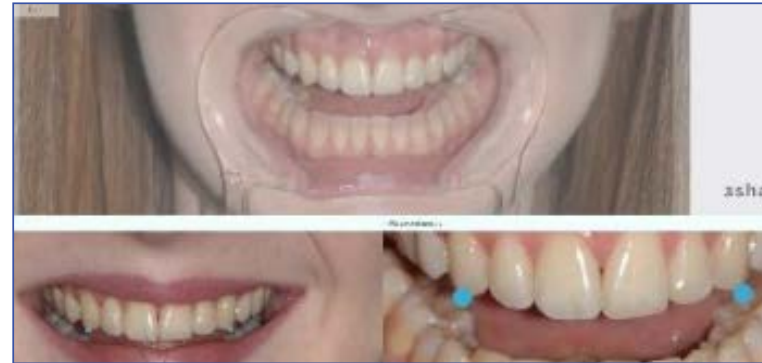

Figure 6. Aligned pictures in smile design program.

shape, which could be optionally altered. As only the incisors were planned to be altered without surgical treatment, the gingival line was replaced to the original position and the smile curve shape was repositioned to the canine-premolar line, the result being that the incisors, mainly the second are shorter than they should be (Fig. 7).

The 2D outline of the teeth was designed and the parameters (texture, color, brightness) of the smile simulation were set. The smile simulation was transferred to the patient's mobile phone through an application (my3Shape app, 3Shape, Copenhagen, Denmark), in which she could see the before and after smile situation in one picture (Fig. 8). Finally, the retracted photo with the outline of the designed teeth was exported from the software, and was used as a guide for a digital wax-up.

\subsection{Digital wax-up}

For a digital wax-up, an intraoral scan was taken from the initial status of the maxillary and mandibular arches and maximum intercuspation (Trios 3 POD, 3Shape, Copenhagen, Denmark). The intraoral scans were sent to the laboratory software (Dental System, 3Shape, Copenhagen, Denmark), in which the scans and the picture with the smile design were aligned on six pairs of points. Then the desired shape of the teeth was designed on the initial scan guided by the outline of the smile design, and a 3D printed model was produced with this design (Figs. 9-10).

\subsection{Mock-up, pre-preparation scan and preparation}

For the 3D printed model (Bego Varseo S, Bremen, Germany), a template was made and a bisacryl resin (Dental Central Crown and Bridge Material, Hannover, Germany) mock-up was fabricated intraorally (Fig. 11). The patient was satisfied with the shape and the new smile, and changes in phonetics were not noticeable. The mock-up was retained by friction and used for guided minimally invasive preparation $[4,5]$. Three calibration points were cut with round diamond burs into the incisal part for 0.4 $\mathrm{mm}$ and two calibration grooves to the vestibular surface for $0.3 \mathrm{~mm}$ reduction (Fig. 12). Then an overall reduction was performed with a chamfer diamond bur. The thickness of the mock-up was $0.1 \mathrm{~mm}$ on average, so the preparation resulted in $0.2-0.3 \mathrm{~mm}$ minimal enamel loss. The margin line was prepared first paragingivally, then by double corded sulcus 


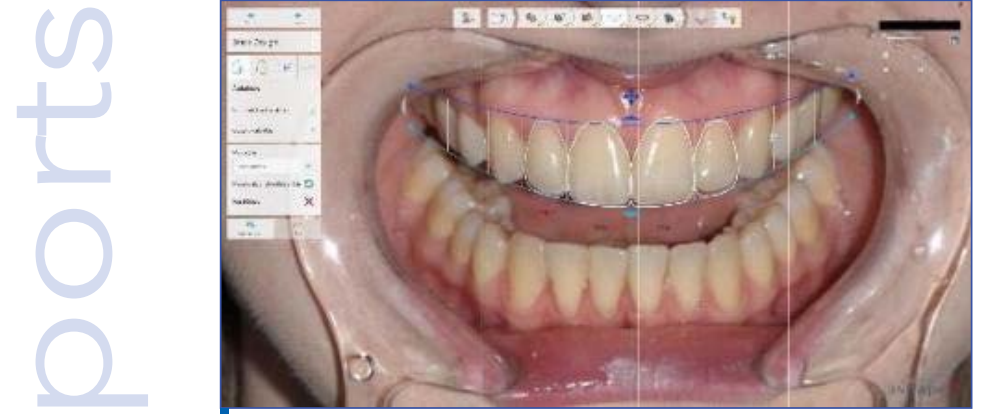

Figure 7. Ideal smile curve shape.

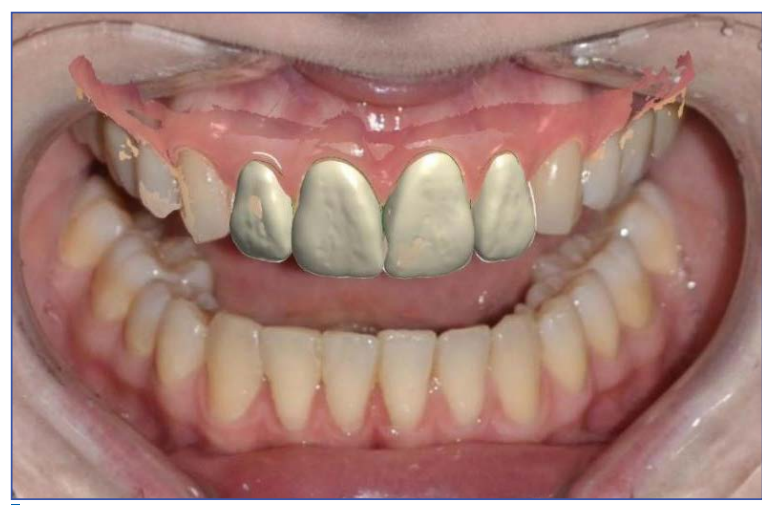

Figure 9. Digital wax-up.

retraction (Ultrapak \#00 and \#000, Ultradent, South Jordan, UT, USA) $0.5 \mathrm{~mm}$ subgingivally (Fig. 13). The enamel of the contact surfaces was reduced with the preparation of the buccal surface, however the remaining contact point was not separated directly. A new workflow was started in the software and a digital impression was made from the maxilla with a mock-up as a pre-preparation scan. For this scan, a second mock-up was fabricated for the prepared teeth (Fig. 14), because it imitated the desired thickness and marginal fit for the final veneers and resulted in a finer shape and surface so later the copy of the pre-preparation scan could perform a more accurate design for the final veneers. The second mock-up was also used as temporary veneers and retained by flow composite resin without etching and bonding.

\subsection{Digital impression}

A digital impression was taken from the prepared teeth by sulcus retraction then from the mandibular arch and maximum intercuspation. The prescription was sent to the laboratory.

\subsection{Shade measurement and material selection}

Before preparation, the right central incisor was selected as a reference tooth for shade selection. $A$ reference photo was taken with a B1 tab chosen as best match (VITA Classical shade guide, VITA Zahnfabrik, Bad Säckingen, Germany). The actual tooth shade was a bit darker than B1; the lightness level could be described with the A1 tab but with the yellow hue of a B tab (Fig. 15). After preparation, a die shade measurement was also performed; the

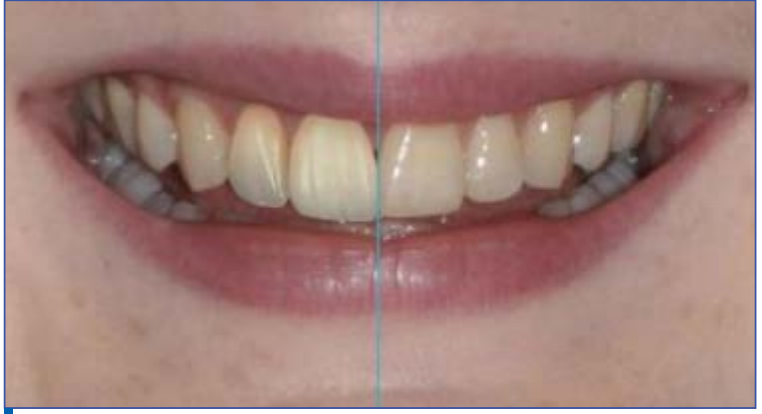

Figure 8. Smile simulation.

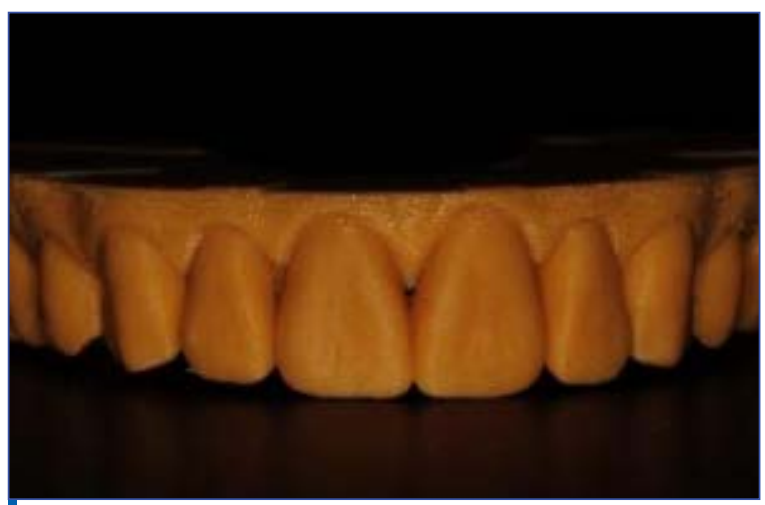

Figure 10. 3D printed model.

color was identified as ND2 (IPS Natural Die Material Shade Guide, Ivoclar Vivadent, Wien, Austria) (Fig. 16). For the $0.3 \mathrm{~mm}$ ultra-thin lithium-disilicate veneers, a medium-translucency B1 color ingot was selected (IPS e.max Press MT B1, Ivoclar Vivadent, Wien, Austria).

\subsection{Laboratory procedure}

The prescription was accepted in the laboratory design software. After margin line and insertion direction determination, the design was made by the morph to pre-preparation function of the software, which resulted in a $0.006 \mathrm{~mm}$ precise copy of the mock-up. This way, the exact same shape that the patient accepted intraorally could be transferred to the final design on the prepared teeth (Fig. 17). As we chose to produce the final restoration from pressed lithium-disilicate, the wax pattern for the pressing technique was performed by milling out the final design of the restoration from a wax block (APWhite Lemon Wax Blank, Aesthetic-Press, Düsseldorf, Germany with Zenotec Mini, Wieland Dental, Pforzheim, Germany). The wax pattern was sprued, invested (IPS PressVEST Premium, Ivoclar Vivadent, Wien, Austria) and fired out (Denkal 4B, Calory Hitech Technical Ltd., Budapest, Hungary), then pressed from the lithium disilicate block (Programat EP 3000, Ivoclar Vivadent, Wien, Austria). After checking the fit of the veneers intraorally (Fig.18), staining and glazing was made in the laboratory on 3D printed model (Fig. 19) with the patient's attendance (IPS Ivocolor Essence and Shade, Glaze Powder FLUO and Mixing Liquid Longlife, Ivoclar Vivadent, Wien, Austria). During staining, the veneers were tried in 


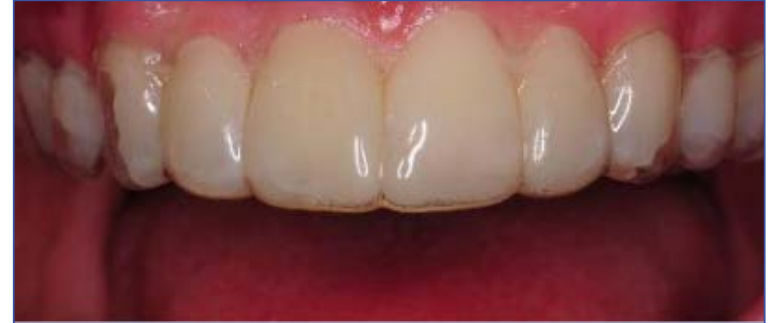

Figure 11. Template and bisacryl resin.

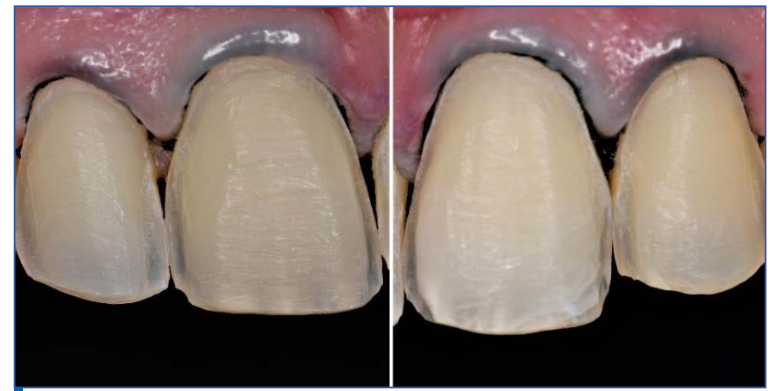

Figure 13. Prepared teeth with minimally-invasive method, sulcus retraction.

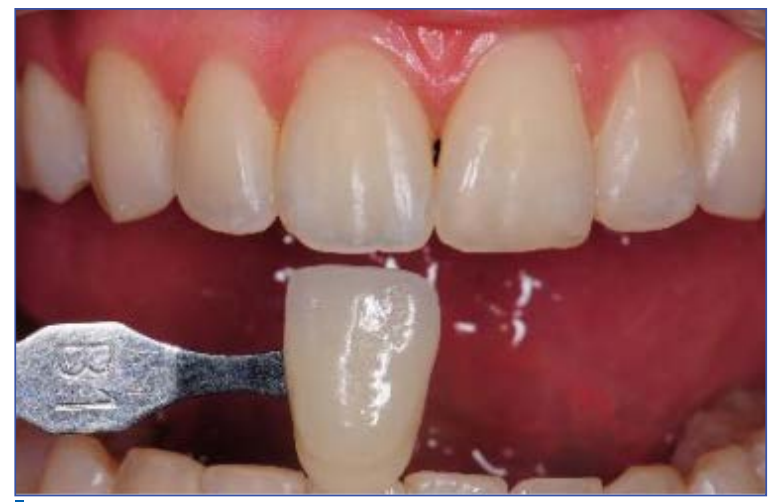

Figure 15. Shade measurement before preparation.

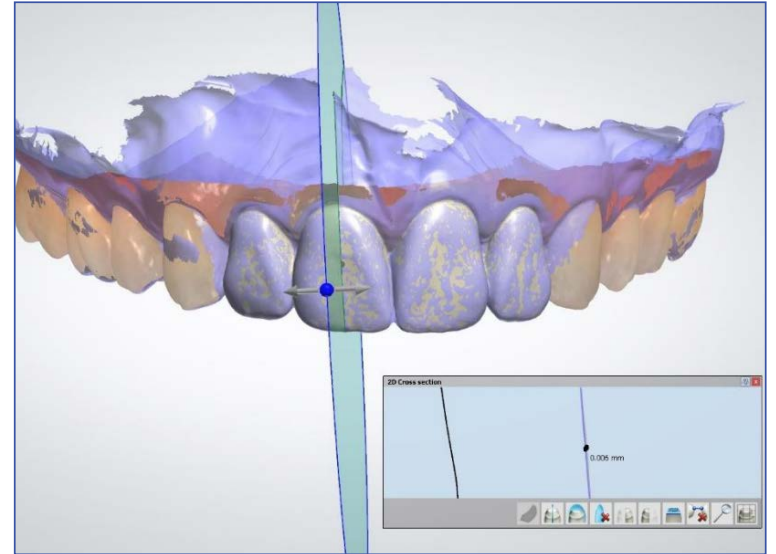

Figure 17. Virtual design of the final restoration.

with try-in paste in neutral color (Variolink Esthetic Try-In Paste Neutral, Ivoclar Vivadent, Wien, Austria)..

\subsection{Cementation}

The cementation protocol was used according to Ivoclar Vivadent's Cementation Navigation System [14]. The surfaces of the veneers were etched and silanated in one step with a primer (Monobond Etch

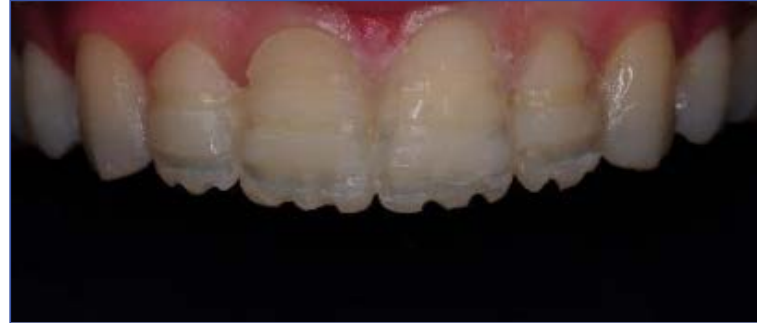

Figure 12. Calibration points and grooves.

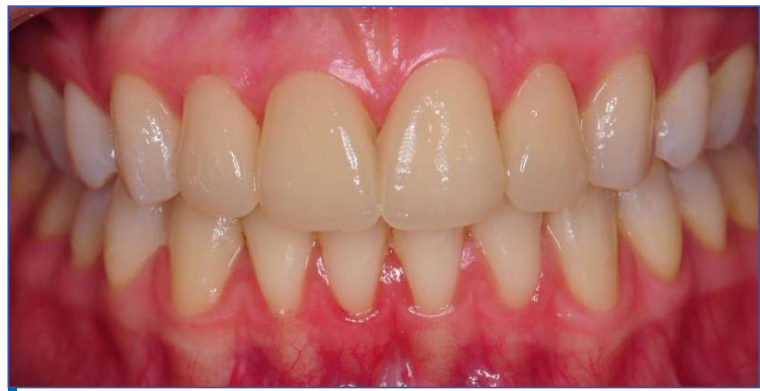

Figure 14. Intraoral mock-up after preparation.

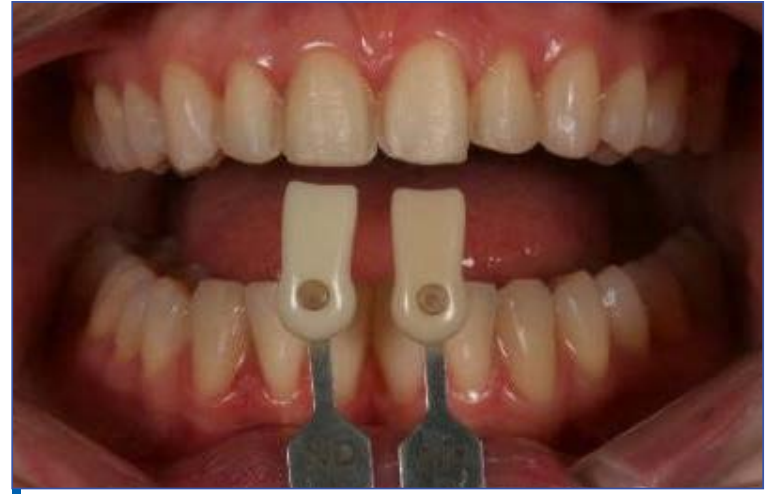

Figure 16. Die shade measurement.

\& Prime, Ivoclar Vivadent, Wien, Austria).

A sulcus retraction cord and rubber dam isolation were applied, and the teeth were prepared with the two-step total etch technique (Ultra-Etch, Ultradent Products, Cologne, Germany and $3 \mathrm{M}$ Adper Single Bond Plus Adhesive, 3M ESPE, St. Paul, MN, USA). A light-curing resin cement in the same neutral color as the try-in paste was applied for the veneers and placed in the right position (Variolink Esthetic LC Neutral, Ivoclar Vivadent, Wien, Austria). The veneers were spot-polymerized for 2 seconds for easy excess cement removal, then fully polymerized for 60 seconds on each surface [5]. The veneers were cemented one by one (Figs. 20-21). After cementation, the occlusal contact points were checked in maximal intercuspation position, by protrusion (Figs. 22-24).

\section{Discussion}

Similar case reports for smile design and ultra-thin veneers were published in the last few years $[1,4,5]$. Digital smile design was performed by designing the shape of the teeth and the gingival line in harmony with the form of the face using definite 

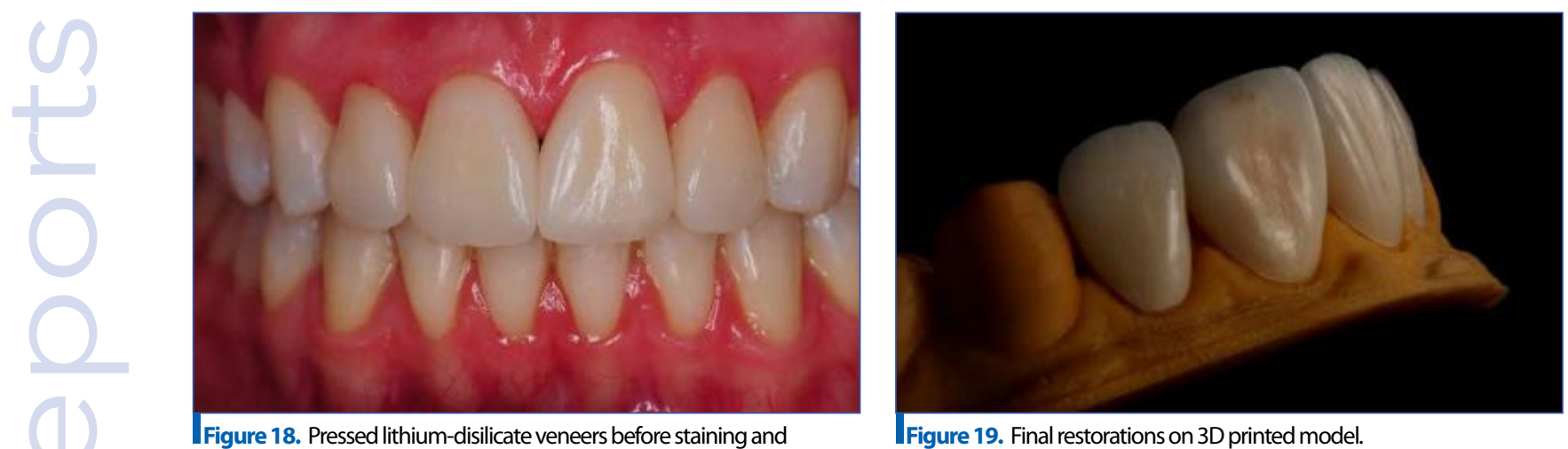

Figure 18. Pressed lithium-disilicate veneers before staining and

Figure 19. Final restorations on 3D printed model.
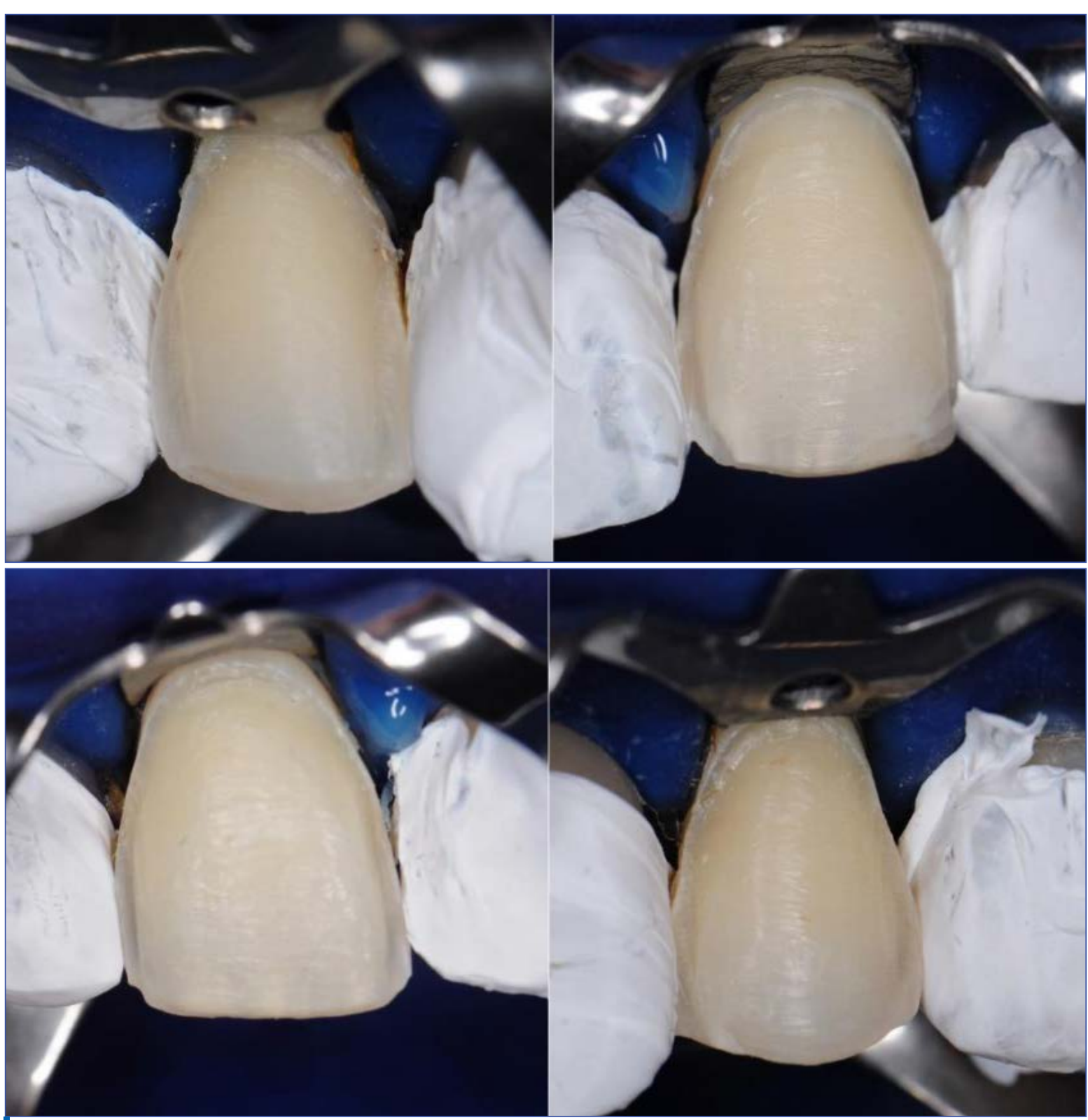

Figure 20. Cementation in absolute isolation

points and reference lines to approve the esthetics of the patient's smile. The impression was taken using the conventional method $[1,4,5]$. Hu et al [1] used the scanned mock-up form as a "ghost image" to guide the design of the final shape, albeit not to a fully anatomical form. A dentin cut-back shape was designed, milled from wax, pressed, and the incisal part was layered, which could lead to a difference between the mock-up and the final shape [1]. Stanley et al [2] described a fully digital workflow for a full-mouth rehabilitation, in which digital smile design and digital impression was performed, and 

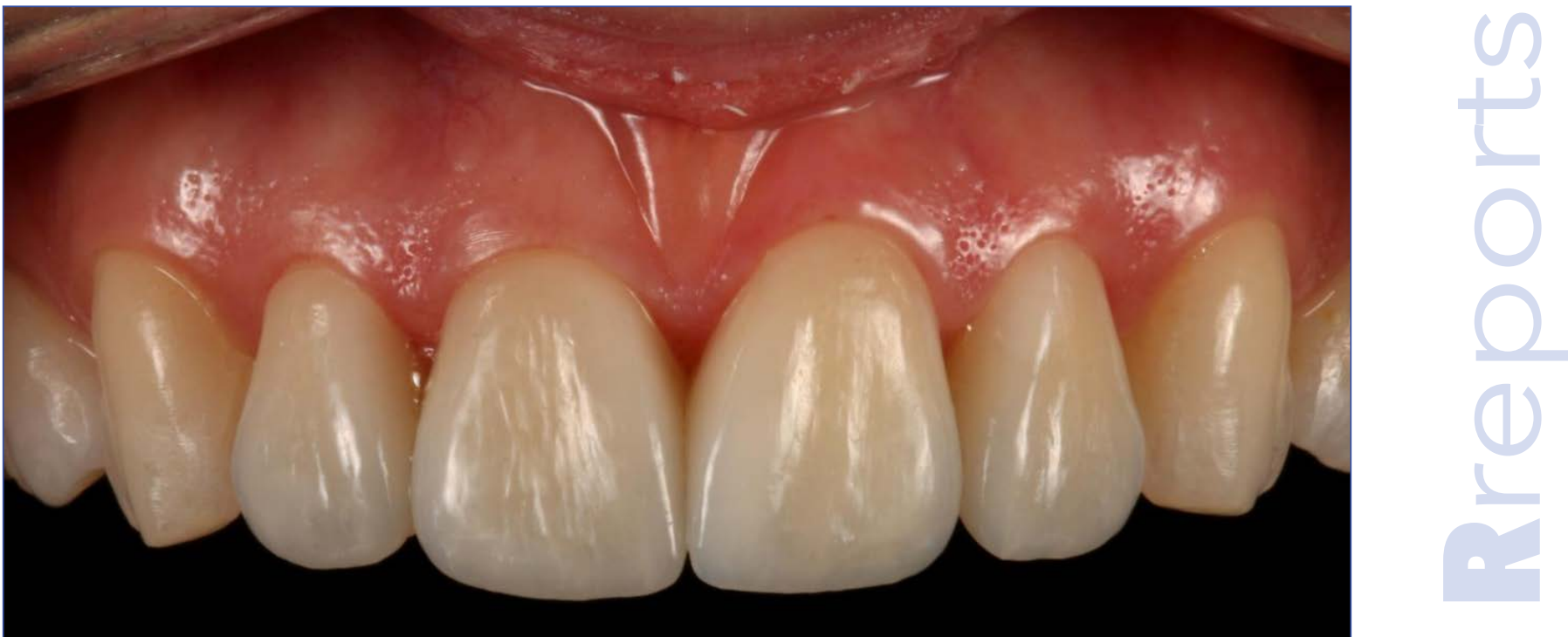

Figure 21. Cemented final restorations.
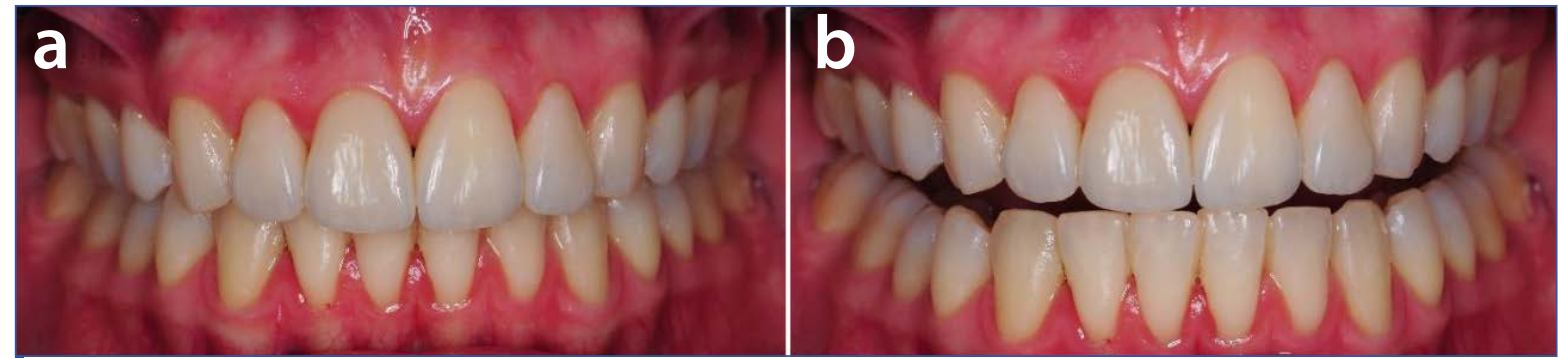

Figure 22. Maximal intercuspation position (a) and protrusion (b).

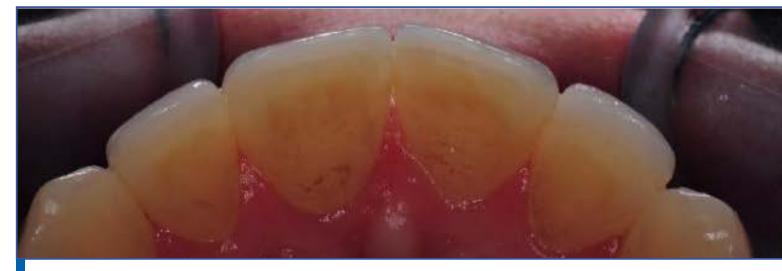

Figure 23. Incisal margin from palatal view.

the digital model of the wax-up was 3D printed. The mock-up was performed with vacuum formed matrix and bysacril; then, after a guided preparation, the definitive veneers were designed computeraided and milled from e.max CAD [2]. However, in this case report it is not mentioned how the shape of the mock-up was transformed for the final restoration. The wax-up - mock-up technique for patient motivation and guided preparation is now a well-known, long used method [5]. The aim of a mock-up driven preparation is to save as much hard tissue as possible. The preparation starts from the desired form with calibration grooves on the vestibular surface; then, with a chamfer bur, the vestibular surface is prepared uniformly in the depth of the calibration grooves. Using this method, the tooth structure is only removed from where it is needed for the final restoration [5]. Non-prep veneers are also considered as a possibility for minimally invasive treatment; however, according to Magne et al [15], the laboratory process and the handling of these veneers are extremely challenging and

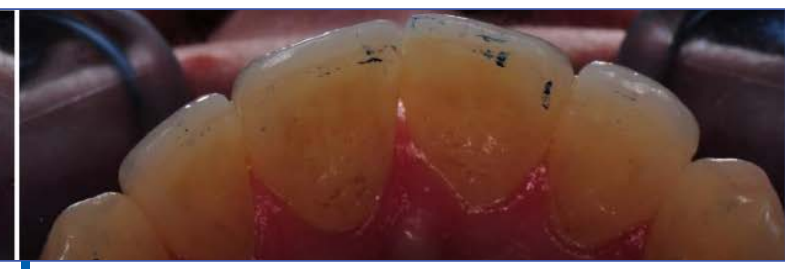

Figure 24. Anterior-guidance.

usually end-up in bulky, unsupported margins with a risk of chipping [15]. Transferring the exact shape of the mock-up to the final restoration is essential. One benefit of the mock-up is that the patient can visualize the new smile and can accept the shape, but if the copy of the shape is not exact to a hundred percent, it could result in conflicts with the patient after inserting the final restoration [5]. Another advantage why a mock-up should be prepared is the guided nature of the preparation, which means that a uniform, minimally invasive preparation and veneer wall-thickness can be achieved. If the design of the veneers is altered relative to the mock-up, a discrepancy from the desired wall-thickness and color may occur [4]. The method described in a case report by Kovács [16] is a possible method to copy the exact same shape of the mock-up to the final veneers. A pre-preparation scan is to be taken from the mock- up in the same workflow with the preparation scan from the abutments, after which the "morph to pre-preparation" function in the laboratory software results in a precise copy [16]. 


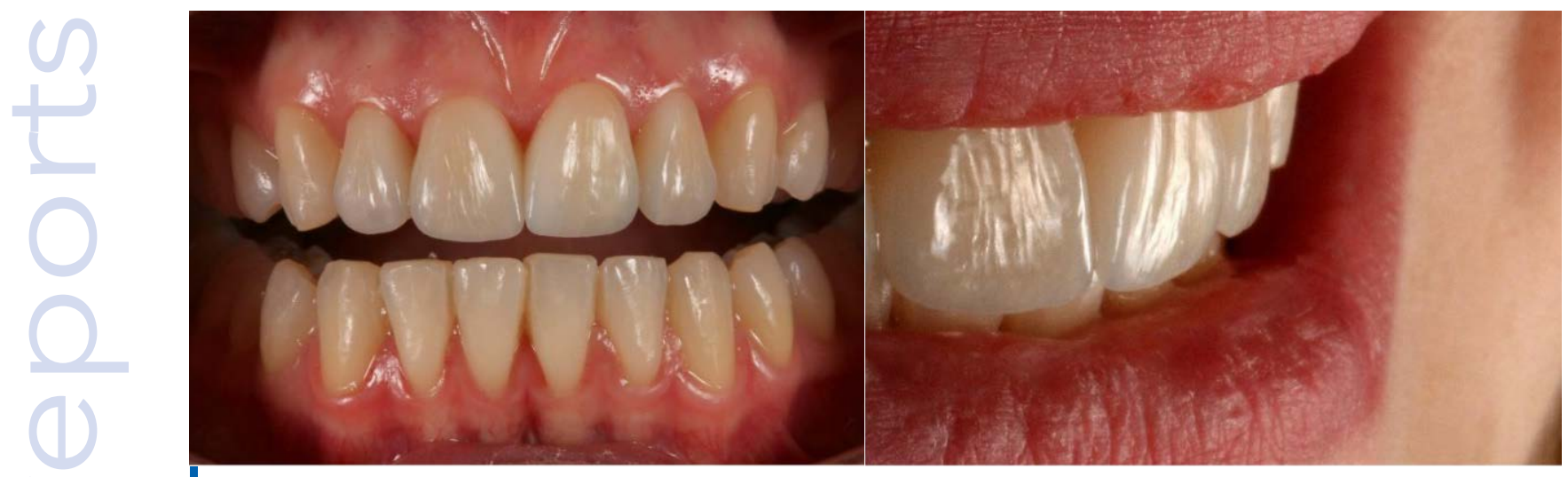

Figure 25. Final result.

The material choice in this case report was based on published in vitro studies, in which the fracture resistance and marginal fit accuracy of IPS e.max Press and CAD were examined [8-12,17]. The length of the silicate crystals in e.max Press is 3-6 $\mu \mathrm{m}[8]$ and in e.max CAD they are 0.1-1 $\mu \mathrm{m}[10]$. The proportion of the crystal phase in Press is 70\% [8]; however, in CAD in the blue phase it is $40 \%$, which will transform after sintering into $70 \%$ [9]. It is suggested that crystallization is more complete in the pressed material than in sintered CAD blocks [10]. Because of the differences in the crystal structure, the fracture toughness, flexural strength and fracture load parameters of e.max Press are greater than those of CAD $[8,9]$. Furthermore, according to Schestatsly et al [10], the milling procedure initiates a cascade of cracks in the material, which results in chipping [10]. These statements correlate with Alkadi et al [11], who describe that the fracture toughness of CAD blocks is lower because of a smaller crystal length and phase, and the fractures formed in the glossy matrix where the crystal transformation was not complete. Regarding the marginal fit of lithium disilicate crowns, those made with the press technique after making a wax pattern by hand had a significantly better accuracy of fit than crowns milled from a CAD block [12]. The reason is suggested to be an easier adaptation of the wax pattern to the dies than to the milled ceramic. According to a critical review, most of the studies comparing the pressed and milled restorations concluded that the marginal fit of e.max Press is more accurate than e.max CAD [17]. The pattern for the pressing technique can be produced by several methods depending on the impression technique and material of the pattern. According to Homsy et al [18], for conventional impressions, lithium-disilicate crowns pressed from milled wax patterns have a more precise marginal fit than those from free-hand wax patterns. In their in vitro study, six combinations of techniques were compared with the following parameters: for conventional impression and gypsum cast making, wax and resin patterns by hand; for conventional impression but digitalized stone dies, milling wax and PMMA patterns; and for digital impressions, milling wax and PMMA patterns. All inlay patterns were pressed from e.max Press lithium-disilicate. It was concluded that the accuracy of the marginal fit depends on the impression technique but is independent from the material of the pattern; however, the internal fit of the restorations depends on the pattern's material.

Overall, the most accurate method was when a wax pattern was milled after digital impression [18]. However, it must be considered that another option to produce patterns with the CAD/CAM technique is $3 \mathrm{D}$ printing. Elfar et al. and Kang et al. compared pressed restorations from free-hand, milled and 3D printed wax patterns, but concluded with different results. According to Elfar et al [19], crowns from 3D printed wax patterns were the most accurate [19]; however, Kang et al [20] reported that veneers from milled wax patterns had the best marginal fit [20]. It has to be mentioned that they produced different types of restorations and different methods to evaluate the results.

Although it is still not conclusive whether the 3D printing or the milling technique is more accurate, it is described in the two studies that both CAD/CAM techniques are more accurate than free-handed approaches, and the marginal fits of all techniques are in the clinically acceptable range $[19,20]$. The cementation of lithium-disilicate pressed veneers was performed with an adhesive technique in absolute rubber dam isolation using light curing resin cement. There are two methods mentioned for surface treatment of the veneers.

The Cementation Navigation System by Ivoclar Vivadent describes a one-component primer called Monobond Etch and Prime for preparing the surface [14]; however, the Scientific Documentation of IPS e.max Press [8] offers another option: first, 5\% hydrofluoric gel (IPS Ceramic Etching Gel), then a silanizing agent called Monobond Plus is to be applied to the surface.

In an in vitro study, pressed lithium-disilicate crowns were produced and cemented with resin cement to epoxy resin-reinforced glass fiber dies with the two different surface treatments mentioned above. The results showed that crowns treated with Monobond Etch \& Prime had greater mechanical structural reliability and higher survival rate than those treated with Monobond Plus [10]. 


\section{Conclusion}

In cases when restorations are prepared in the esthetic region, the communication between the patient and the dentist is always the first key to success. The patient has to understand the opportunities and limitations, while the dentist has to get to know the expectations of the patient. An ideal situation to communicate is when the form of the restorations can be visualized in the mouth by performing a mock-up.

Digital devices and software offer an effective way for precise, time efficient, predictable, comfortable, and individually designed rehabilitation of the patient's smile; however, in spite of the potential offered by fully digital workflows, in some cases, considering conventional laboratory procedures such as pressing lithium-disilicate produces a better outcome (Fig. 25).

\section{Author Contributions}

MD: performed clinical procedure, wrote initial manuscript, AB: performed laboratory procedure and final result photos, ZK: conception and design of the study, JB: revised the article critically for important intellectual content, $\mathrm{PH}$ : approved the final version to be submitted.

\section{Acknowledgments}

The authors thank for Dental-Trade Kft., Budapest, Hungary for producing 3D printed models.

\section{References}

1. Hu JC, Klimmek K. Minimal Veneer Intervention for Maximal Smile Transformation. J Cosmet Dent. 2018;34(3):28-37.

[Full text link] Google Scholar

2. Stanley M, Paz AG, Miguel I, Coachman C. Fully digital workflow, integrating dental scan, smile design and CAD-CAM: case report. BMC Oral Health. 2018;18(1):134. [CrossRef] [free PMC article] [PudMed] Google Scholar Scopus

3. Coachman C, Paravina RD. Digitally Enhanced Esthetic Dentistry - From Treatment Planning to Quality Control. J Esthet Restor Dent. 2016;28:S3-S4.

[CrossRef] [PubMed] Google Scholar Scopus

4. Coachman C, Gurel G, Calamita $M$, et al. The Influence of Tooth Color on Preparation Design for Laminate Veneers from a Minimally Invasive Perspective: Case Report. Int J Periodontics Restorative Dent. 2014;34(4):453-459.

[CrossRef] [PubMed] Google Scholar Scopus

5. Gürel G. Predictable and precise tooth preparation techniques for porcelain laminate veneers in complex cases. Int Dent SA. 2007;9(1):30-40.

[Full text link] Google Scholar

6. Smielak B. No-Preparation and Minimally Invasive Veneers in Clinical Practice : Part 1. Smile Dent J. 2015;10(1):12-15.

[CrossRef] Google Scholar

7. IPS e.max ${ }^{\circledR}$ Clinical Guide [Internet]. 2013 [cited 10.25.2019.] Available from: https://www.ivoclarvivadent.ru/zoolu-website/ media/document/1269/IPS+e-max+Clinical+Guide.

8. Bühler-Zemp P, Völkel T, Fischer K. IPS e.max ${ }^{\circledR}$ Press Scientific Documentation [Internet]. 2011 [updated 2011 March; cited 10.25.2019.] Available from: https://www.ivoclarvivadent.fr/ zoolu-website/media/document/9808/IPS+e-max+Press.

9. Fischer $K$, Bühler-Zemp $P$, Völkel T. IPS e.max ${ }^{\circledR}$ CAD Scientific Documentation [Internet]. 2011 [updated 2011 March; cited 10.25.2019.] Available from: https://www.ivoclarvivadent.com/ zoolu-website/media/document/9793/IPS+e-max+CAD.

10. Schestatsky R, Zucuni CP, Venturini AB, et al. CAD-CAM milled versus pressed lithium-disilicate monolithic crowns adhesively cemented after distinct surface treatments: Fatigue performance and ceramic surface characteristics. J Mech Behav Biomed Mater. 2019;94:144-154.

[CrossRef] [PubMed] Google Scholar Scopus

11. Alkadi L, Ruse ND. Fracture toughness of two lithium disilicate dental glass ceramics. J Prosthet Dent. 2016;116(4):591-596.

[CrossRef] [PudMed] Google Scholar Scopus

12. Azar B, Eckert S, Kunkela J, Ingr T, Mounajjed R. The marginal fit of lithium disilicate crowns: Press vs. CAD/CAM. Braz Oral Res. 2018;32(0).

[CrossRef] [PubMed] Google Scholar

13. Mangano F, Gandolfi A, Luongo G, Logozzo S. Intraoral scanners in dentistry: a review of the current literature. BMC Oral Health. 2017:17(1):149.

[CrossRef] [free PMC article] [PubMed] Google Scholar

14. Cementation Navigation System ${ }^{\circledR}$ Ivoclar Vivadent [Internet] 2019.http://www.cementation-navigation.com/en/a/tooth/ veneer/lithium-disilicate/variolink-esthetic/adhese-universal.pdf. 15. Magne $P$, Hannah J, Magne M. The case for moderate guided prep indirect porcelain veneers in the anterior dentition. The pendulum of porcelain veneer preparations: from almost nonprep to over-prep to non-prep. Eur J Esthet Dent. 2013;8(3):376-388. [Full text link] [PubMed] Google Scholar

16. Kovács ZI, Borbely J Schmidt P, Hermann P. CAD/CAM Monolithic Restoration in Molar Region without Occlusal Adjustment. Poster No 38 presented. In: Digital Dentistry Society Global Conference. October 3-5,2019; Baden-Baden, Germany.

17. Mounajjed R, Layton DM, Azar B. The marginal fit of E.max Press and E.max CAD lithium disilicate restorations: A critical review. Dent Mater J. 2016;35(6):835-844. [CrossRef] [PubMed] Google Scholar Scopus

18. Homsy FR, Özcan M, Khoury M, Majzoub ZAK. Comparison of fit accuracy of pressed lithium disilicate inlays fabricated from wax or resin patterns with conventional and CAD-CAM technologies. $J$ Prosthet Dent. 2018;120(4):530-536.

[CrossRef] [PubMed] Google Scholar Scopus

19. Elfar M, Korsel A, Kamel M. Marginal fit of heat pressed lithium disilicate crowns fabricated by three-dimensional printed and subtractive CAD/CAM wax patterns. Tanta Dent J. 2018;15(4):199. [CrossRef] [Full text link] Google Scholar

20. Kang S-Y, Lee H-N, Kim J-H, Kim W-C. Evaluation of marginal discrepancy of pressable ceramic veneer fabricated using CAD/ CAM system: Additive and subtractive manufacturing. J Adv Prosthodont. 2018;10(5):347.

[CrossRef] [free PMC article] [PubMed] Google Scholar Scopus 


\section{Mariann DANKÓ \\ DMD, Clinical Doctor, Resident Department of Prosthodontics Faculty of Dentistry Semmelweis University Budapest, Hungary}

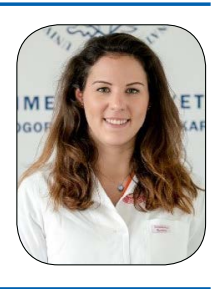

Dr. Mariann Dankó, DMD, graduated from Faculty of Dentistry, Semmelweis University, Budapest, Hungary in 2018. She is currently attending the residency program in Prosthodontics at the Department of Prosthodontics, Faculty of Dentistry, Semmelweis University, Budapest, Hungary. She participates in the Digital Dentistry, and Implant Prosthetics Working Groups. She works with digital appliances and performs digital workflows also in private practice.

\section{Ouestions}

\section{What is/are the advantage(s) of a fully digital workflow?}

口a. Time-efficiency;

b. Simplified clinical procedures;

ac. Less discomfort for the patient;

$\square$ d. All of them

\section{Which type of pattern was used to press lithium-disilicate veneers in this study?}

Da. Milled wax pattern;

ab. 3D printed wax pattern;

ac. Hand-crafted wax pattern;

$\square$ d. Hand-crafted resin pattern.

\section{Why is it considered to get back to a conventional pressing method while performing a digital workflow?}

Da. Pressed lithium-disilicate has more accurate marginal fit;

ab. Milled lithium-disilicate has better physical properties;

uc. Pressing lithium-disilicate is a faster procedure than milling;

ad. All of them.

\section{Why is it important to perform an intraoral mock-up for the initial status?}

$\square$ a. Facilitates the communication with the patient and laboratory;

ub. Possible to adjust according to patient's expectation;

uc. Offers a guided minimally invasive preparation option for preserving hard tissue;

$\square$ d. All of them.

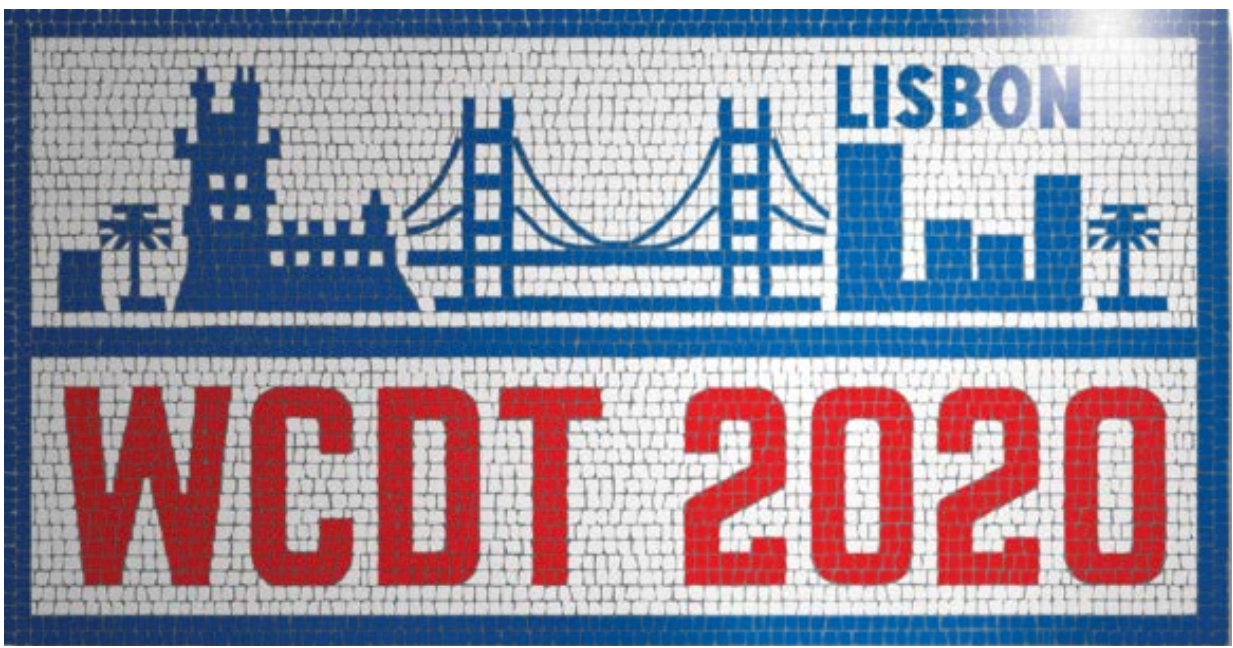

21 st

World Congress

on Dental

Traumatology

Lisbon, Portugal

17-20 th June 